\title{
Superradiance, bosonic Peierls distortion, and lattice gauge theory in a generalized Rabi-Hubbard chain
}

\author{
Axel Gagge (1) and Jonas Larson \\ Department of Physics, Stockholm University, Se-106 91 Stockholm, Sweden
}

(Received 30 June 2020; accepted 17 November 2020; published 11 December 2020)

\begin{abstract}
We investigate a one-dimensional Rabi-Hubbard type of model, arranged such that a quantum dot is sandwiched between every cavity. The role of the quantum dot is twofold, to transmit photons between neighboring cavities and simultaneously act as an effective photon nonlinearity. We consider three-level quantum dots in the $\Lambda$ configuration, where the left and right leg couple exclusively to the left or right cavity. This noncommuting interaction leads to two highly entangled incompressible phases, separated by a second-order quantum phase transition; the degrees of freedom of the quantum dots can be viewed as a dynamical lattice for the photons which spontaneously breaks $\mathbb{Z}_{2}$ symmetry due to a bosonic Peierls instability, leading to a phase with dimerized order. Additionally, we find a normal insulating phase and a superfluid phase that acts as a quantum many-body superradiant phase. In the superradiant phase, a $\mathbb{Z}_{2}$ symmetry is broken and the phase transition falls within the universality class of the transverse-field Ising model. Finally, we show that the model can be interpreted as a $\mathbb{Z}_{2}$ lattice gauge theory in the absence of a dipolar field on the lower qutrit levels.
\end{abstract}

DOI: 10.1103/PhysRevA.102.063711

\section{INTRODUCTION}

At the quantum level of single photons, strongly coupled light and matter can form novel states with no counterparts in other branches in physics [1]; new interacting quantum manybody models arise which may host exotic phases. The study of phase transitions (PTs) in light-matter systems dates back to the early days of the laser, when the onset of lasing with increasing pump power was identified as a nonequilibrium continuous PT [2].

Predating the laser, in 1954 Dicke showed how the rate of spontaneous emission for a set of $N$ two-level atoms could be enhanced by a factor $\sqrt{N}$ [3]. This phenomenon, arising due to collective multipartite interference, has been termed superradiance and can be derived from the Dicke model describing the coupling of $N$ identical two-level systems with a single-photon mode [4]. In 1973, first by Hepp and Lieb [5] and shortly afterwards by Wang and Hioe [6], it was demonstrated that the Dicke model supports a second-order PT from a "normal" to a "superradiant" phase as the light-matter coupling is raised above a critical value. The corresponding PT is accompanied by a spontaneous breaking of a $\mathbb{Z}_{2}$ symmetry. In more recent times, there has been a strong interest in realizing PTs, like the Dicke one, in quantum optical lattice systems [7]. This development was spurred by the increased experimental

Published by the American Physical Society under the terms of the Creative Commons Attribution 4.0 International license. Further distribution of this work must maintain attribution to the author(s) and the published article's title, journal citation, and DOI. Funded by Bibsam. control over many-body quantum systems and in the wake of quantum simulators [8].

One manifestation of a quantum PT (QPT), i.e., a transition driven by quantum rather than thermal fluctuations, is a nonanalytic behavior of the system's ground state [9]. For the Dicke PT one finds such a nonanalyticity, and as such it has often been referred to as a QPT. Nevertheless, the transition is of a mean-field type, and the role of quantum fluctuations becomes irrelevant in the thermodynamic limit [10]. In this strict sense, the Dicke PT is not a proper QPT. In a truly quantum normal-superradiant PT, quantum fluctuations should remain extensive in the thermodynamic limit, as the system approaches the superradiant transition. This is more in the vein of paradigmatic quantum critical models like the transverse-field Ising or Bose-Hubbard models [11]. One way to enhance the role of quantum fluctuations is to consider multimode Dicke models [12] or cavity arrays [13]. In such models, arrays of cavities or resonators are constructed on microchips such that photons can tunnel between neighboring cavities. Each transmission-line resonator is equipped with a quantum dot (q-dot) that acts as an artificial two-level atom [14]. This produces a Jaynes-Cummings nonlinearity [15], which serves as an effective photon-photon interaction. When photon losses can be neglected, the effective excitations are polaritons governed by a Hamiltonian similar to the BoseHubbard model. Similarly, superfluid and insulating phases of polaritons appear [16].

In [17], the light-matter terms of the model were used to produce an effective interaction as well as the kinematics. The authors studied a one-dimensional array of resonators, with a two-level system (qubit) placed between neighboring resonators, such that photon tunneling is mediated by a Rabi-type interaction. It was demonstrated that the low-energy physics is described by the transverse-field Ising model. While not 
(a)

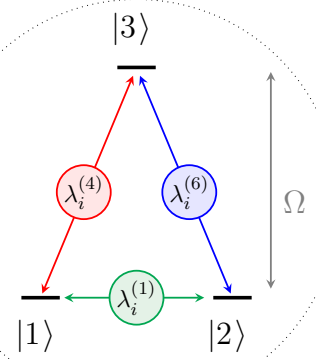

(b)

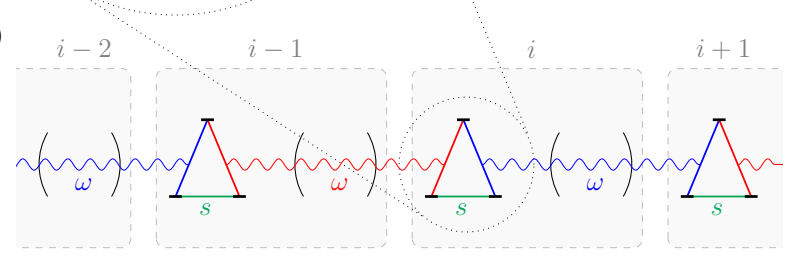

FIG. 1. Figure (a) shows a schematic picture of a qutrit in the $\Lambda$ configuration. The operators $\lambda^{(1)}, \lambda^{(4)}$, and $\lambda^{(6)}$ act like Pauli $\sigma^{x}$ matrices between each pair of qutrit levels. Figure (b) displays how these qutrits are connected with cavities in our model. Each cavity supports a single mode, with frequency $\omega$. Each qutrit has two degenerate bare ground states $|1\rangle$ and $|2\rangle$, and an excited state $|3\rangle$ separated in energy by $\Omega$ [see (a)]. Resonators of odd index couple to the $\lambda^{(4)}$ operator of its adjacent qutrits (shown in red). Resonators of even index instead couple to the $\lambda^{(6)}$ operator of its adjacent qutrits (shown in blue). In addition, there is a direct coupling between the $|1\rangle$ and $|2\rangle$ states with an amplitude $s$ (shown in green).

discussed in Ref. [17], we may think of the emerging Ising transition as a normal-superradiant PT, driven by quantum fluctuations. The idea of interconnecting cavities via superconducting qubits dates back to the works of Ref. [18], where the qubits were considered as controllable switches for the tunneling of photons between the cavities. Based on these ideas, it was possible to experimentally connect three or more resonators with the help of qubits [19].

In this paper we also consider a one-dimensional closed cavity array with mediated photon tunneling. While photon losses are inevitable in cavity arrays, the properties of the corresponding closed system can reveal vital information about the open system $[10,20]$, which motivates our paper. Our model differs from that of Ref. [17] in two important aspects. First, instead of qubits we consider three-level systems, i.e., qutrits with three internal states $|1\rangle,|2\rangle$, and $|3\rangle$. Second, resonators of odd index couple only to the $|1\rangle \leftrightarrow|3\rangle$ transitions of its adjacent qutrits, while resonators of even index instead address only the $|2\rangle \leftrightarrow|3\rangle$ transitions of its adjacent qutrits (see Fig. 1). These modifications lead to the appearance of novel exotic phases in our model.

It may be helpful to picture the photons as "site variables" and the qutrits as "bond variables," forming a "dynamical lattice" for the photons as in quantum link models [21]. A salient result that may arise from a dynamical lattice is an altered periodicity, leading to novel phases such as charge-density waves [22] first predicted by Peierls in one dimension [23], and supersolids [24]. Indeed, for weak coupling strengths we find a bosonic Peierls (BP) phase with altered periodicity. Contrary to a traditional Peierls instability for free fermions, the periodicity is not set by the filling but is induced by effective interactions between the photons. This is akin to the bosonic Peierls transition recently discussed in an extended Bose-Hubbard model with a similar dynamical lattice as the one considered here [25]. Note that the photon number is not a conserved charge in our problem-which is why we refrain from calling the bosonic Peierls phase a charge-density wave despite great similarities. For stronger light-matter couplings, the system undergoes a QPT and is restored to a symmetric phase with a constant, sizable entanglement entropy over the chain [see Eq. (22)].

In order to realize the aforementioned many-body normalsuperradiant QPT we assume a static dipolar field driving the $|1\rangle \leftrightarrow|2\rangle$ transitions. Such a field has a "resetting" effect on the qutrits; e.g., a qutrit undergoing the transitions $|1\rangle \rightarrow|3\rangle \rightarrow|2\rangle$ by transferring one photon between two resonators can be reset by the field to its original state $|1\rangle$ without involving further photons from the resonators. When this field becomes appreciable, two new phases appear in our model. For weak light-matter coupling $g$, a normal symmetric phase (N) appears, characterized by unbroken translational symmetry. For strong values of $g$ we find a superradiant (SR) phase which spontaneously breaks the $\mathbb{Z}_{2}$ parity symmetry that is connected to the superradiant PT.

The outline of the paper is as follows. In the next section we introduce our model and find its symmetries. In Sec. III we present the zero-temperature phase diagram as obtained from density-matrix renormalization-group (DMRG) calculations, and characterize the different phases. We also determine the possible transitions between the phases, and in Sec. IV we give a discussion about physical relevance of our model. Finally, in Sec. V we conclude with a summary.

\section{MODEL SYSTEM AND ANALYSIS}

\section{A. Full Hamiltonian}

We assume ideal qutrits (three-level systems) in the $\Lambda$ configuration: the two states $|1\rangle$ and $|2\rangle$ are degenerate, while the state $|3\rangle$ is of a higher energy. We assume that all transitions can be addressed and coupled to resonators individually. We will suggest methods of experimental realization in Sec. IV. The level configuration and system parameters are shown in Fig. 1(b).

The full model system, as shown in Fig. 1(a), consists of an infinite chain of cavities interspersed with qutrits. We label each qutrit and the resonator to its right by the same site index $i$. Resonators of odd index couple with a Rabi interaction of strength $g$ to the $|1\rangle \leftrightarrow|3\rangle$ transitions of its adjacent qutrits, while resonators of even index instead have a Rabi interaction of the same strength with the $|2\rangle \leftrightarrow|3\rangle$ transitions of its adjacent qutrits [26]. As mentioned in the Introduction, we include a static dipolar field between the two lower qutrit states $|1\rangle$ and $|2\rangle$. Note that such a field breaks the angular momentum conservation if the qutrit is realized with spin quantum numbers, which is possible to realize by external two-photon driving [27]. We do not restrict the analysis to moderate couplings $g \ll \omega$, in which the rotating-wave approximation is applicable, but allow for $g \sim \omega$, i.e., the deep strong-coupling regime [28]. 
The Hamiltonian can be split in a bare and an interaction part:

$$
H=H_{\mathrm{B}}+H_{\text {int }} .
$$

The bare Hamiltonian is composed of the harmonic oscillators representing the single light modes of the resonators, the bare energies of the qutrits, as well as the dipolar field on the two lower qutrit states. Expressed in terms of Gell-Mann matrices [29] (see Appendix A for the definition of the Gell-Mann matrices $\left.\lambda_{i}^{(\alpha)}\right)$, the bare Hamiltonian reads ( $\hbar=1$ throughout)

$$
H_{\mathrm{B}}=\omega \sum_{i} a_{i}^{\dagger} a_{i}-\frac{\Omega}{\sqrt{3}} \sum_{i} \lambda_{i}^{(8)}-s \sum_{i} \lambda_{i}^{(1)},
$$

where $\omega$ is the resonant angular frequency of the cavities, $\Omega$ is the frequency (energy) of the upper qutrit states, $s$ is the strength of the dipolar field on the lower qutrit states, and $a_{i}^{\dagger}\left(a_{i}\right)$ is the photon creation (annihilation) operator for the $i$ th cavity; i.e., for a photon Fock state of the $i$ th cavity, $a_{i}|n\rangle_{i}=\sqrt{n_{i}}|n-1\rangle_{i}, a_{i}^{\dagger}|n\rangle_{i}=\sqrt{n_{i}+1}|n+1\rangle_{i}$, and for the number operator $n_{i}|n\rangle_{i}=a_{i}^{\dagger} a_{i}|n\rangle_{i}=n_{i}|n\rangle_{i}$. The interaction Hamiltonian can be written as

$$
\begin{aligned}
H_{\text {int }}= & g \sum_{i \text { odd }}\left(a_{i}+a_{i}^{\dagger}\right)\left(\lambda_{i-1}^{(4)}+\lambda_{i}^{(4)}\right) \\
& +g \sum_{i \text { even }}\left(a_{i}+a_{i}^{\dagger}\right)\left(\lambda_{i-1}^{(6)}+\lambda_{i}^{(6)}\right),
\end{aligned}
$$

where $g$ is the Rabi coupling strength. Note that the operators $\lambda_{i}^{(4)}$ and $\lambda_{i}^{(6)}$ are proportional to dipole operators for the $|1\rangle \leftrightarrow|3\rangle$ and $|2\rangle \leftrightarrow|3\rangle$ transitions, respectively, and act like $\sigma_{x}$ Pauli matrices for these transitions. Since $\left[\lambda^{(4)}, \lambda^{(6)}\right] \neq 0$, the individual interaction terms do not commute. Also, note that it is possible to reformulate the Hamiltonian with spin-1 operator matrices [30], $S_{\alpha}(\alpha=x, y, z)$, but the price one pays is that it will contain higher-order terms, e.g., $\lambda^{(4)}=S_{x}^{2}-S_{y}^{2}$.

There is a duality which maps the Hamiltonian $H(s, g, \omega, \Omega) \rightarrow H(-s, g, \omega, \Omega)$. The unitary transformation is

$$
U_{D}=\exp \left[i \pi \sum_{i}\left(|1\rangle_{i}\left\langle\left. 1\right|_{i}+\frac{1-(-1)^{i}}{2} n_{i}\right)\right] .\right.
$$

Hence, it is sufficient to consider $s>0$, and we remark that for any superradiant phase for $s<0$ there exists a corresponding superradiant phase for $s>0$, but with a staggered ordered parameter $\left\langle a_{i}\right\rangle \propto(-1)^{i}$.

\section{B. The normal-superradiant phase transition}

Our Hamiltonian is invariant under a $\pi$ rotation with respect to the total excitation number

$$
N_{\mathrm{ex}}=\sum_{i}\left(n_{i}+\sqrt{3} \lambda_{i}^{(8)}\right),
$$

with the corresponding unitary

$$
\Pi=\exp \left(-i \pi N_{\mathrm{ex}}\right)=(-1)^{N_{\mathrm{ex}}} .
$$

Since the eigenvalues of $N_{\text {ex }}$ are integers, we clearly have $\Pi^{2}=\mathbb{I}$ as required for a $\mathbb{Z}_{2}$ symmetry. That the Hamiltonian is symmetric under the action of $\Pi$ follows from noticing

$$
\begin{aligned}
\Pi a_{i} \Pi^{\dagger} & =-a_{i}, \quad \Pi \lambda_{i}^{(4)} \Pi^{\dagger}=-\lambda_{i}^{(4)}, \\
\Pi \lambda_{i}^{(6)} \Pi^{\dagger} & =-\lambda_{i}^{(6)}, \quad \Pi \lambda_{i}^{(8)} \Pi^{\dagger}=\lambda_{i}^{(8)} .
\end{aligned}
$$

Apart from a few special exceptions, continuous phase transitions occur when the ground state of a system spontaneously breaks the symmetries of its Hamiltonian [31]. We can then find a (local) order parameter, which is zero in the symmetric phase and nonzero in the symmetry-broken phase. For the normal-superradiant PT, which is connected to the breaking of a similar $\mathbb{Z}_{2}$ symmetry, a proper choice is $\phi=\langle a\rangle$ [32]. Given a real light-matter coupling $g$, the order parameter will be real; the sign of $\phi$ determines the parity [10]. Similarly in our model, for $s>0$, breaking of the parity symmetry results in a real nonzero value of

$$
\phi \equiv \frac{1}{L} \sum_{i}\left\langle a_{i}\right\rangle
$$

Because of the duality (4), a similar but staggered order appears for $s<0$. The order parameter is the same, except that each term in $(8)$ has to be multiplied by $(-1)^{i}$. Since the symmetry $\Pi$ involves both qutrits and resonators, there are two qutrit order parameters which should show the same (anti)-ferromagnetic order:

$$
\phi_{4 / 6} \equiv \frac{1}{L} \sum_{i}\left\langle\lambda_{i}^{(4 / 6)}\right\rangle
$$

where, again, for $s<0$ each term in (9) has to be multiplied by $(-1)^{i}$.

As a one-dimensional spin chain supporting a $\mathbb{Z}_{2}$ symmetry, the critical point should belong to the universality class of the transverse-field Ising model. To elucidate this, we investigate the limit of $s \rightarrow \infty$ and extrapolate to finite $s$. By a Hadamard transformation of the qutrit states

$$
\begin{aligned}
& |1\rangle_{i} \rightarrow\left(|1\rangle_{i}+|2\rangle_{i}\right) / \sqrt{2}, \\
& |2\rangle_{i} \rightarrow\left(|1\rangle_{i}-|2\rangle_{i}\right) / \sqrt{2}, \\
& |3\rangle_{i} \rightarrow|3\rangle_{i},
\end{aligned}
$$

the Hamiltonian can be rewritten as

$$
\begin{aligned}
H_{\mathrm{B}}^{\prime}= & \omega \sum_{i} a_{i}^{\dagger} a_{i}-\frac{\Omega}{\sqrt{3}} \sum_{i} \lambda_{i}^{(8)}-s \sum_{i} \lambda_{i}^{(3)}, \\
H_{\mathrm{int}}^{\prime}= & g \sum_{i \text { odd }}\left(a_{i}+a_{i}^{\dagger}\right)\left(\tau_{i-1}^{(+)}+\tau_{i}^{(+)}\right) \\
& +g \sum_{i \text { even }}\left(a_{i}+a_{i}^{\dagger}\right)\left(\tau_{i-1}^{(-)}+\tau_{i}^{(-)}\right),
\end{aligned}
$$

where

$$
\tau_{i}^{( \pm)} \equiv \frac{\lambda_{i}^{(4)} \pm \lambda_{i}^{(6)}}{\sqrt{2}}
$$

The bare Hamiltonian has eigenvalues

$$
E_{1 n}=s+\omega n, \quad E_{2 n}=-s+\omega n, \quad E_{3 n}=\Omega+n .
$$

The population in the state with bare energy $E_{2 n}$ will be small if $s$ is large enough and the mean photon number $\bar{n}$ of each resonator is small. In this limit, we project the qutrit degrees 
of freedom of the Hamiltonian by $P=\prod_{i}\left(|1\rangle_{i}\left\langle\left. 1\right|_{i}+\mid 3\right\rangle_{i}\left\langle\left. 3\right|_{i}\right)\right.$ to find

$$
\begin{aligned}
P H_{\mathrm{B}}^{\prime} P & =\omega \sum_{i} a_{i}^{\dagger} a_{i}-\frac{\sqrt{3} \Omega}{2} \sum_{i} \sigma_{i}^{z}+\text { const, } \\
P H_{\mathrm{int}}^{\prime} P & =\frac{g}{\sqrt{2}} \sum_{i}\left(a_{i}+a_{i}^{\dagger}\right)\left(\sigma_{i}^{x}+\sigma_{i-1}^{x}\right),
\end{aligned}
$$

where $\sigma_{i}^{z}=|1\rangle_{i}\left\langle\left. 1\right|_{i}-\mid 3\right\rangle_{i}\left\langle\left. 3\right|_{i} \text { and } \sigma_{i}^{x}=\mid 1\right\rangle_{i}\left\langle\left. 3\right|_{i}+\mid 3\right\rangle_{i}\left\langle\left. 1\right|_{i}\right.$. In this limit, the model is equivalent to that considered in Ref. [17], where it was already concluded that the phase transition falls within the Ising universality class. By the duality (4), we conclude that the Hamiltonian is "antiferromagnetic" for $s<0$.

\section{Quasitranslation symmetry}

Assuming periodic boundary conditions, there is a second global symmetry of the model which we call quasitranslation symmetry: a permutation of the qutrit states $|1\rangle,|2\rangle$, followed by a translation by one site. Calling it $\tilde{\mathcal{T}}$, its action on the operators is

$$
\begin{aligned}
\tilde{\mathcal{T}} a_{i} \tilde{\mathcal{T}}^{\dagger}=a_{i+1}, & \tilde{\mathcal{T}} \lambda_{i}^{(3)} \tilde{\mathcal{T}}^{\dagger}=-\lambda_{i+1}^{(3)}, \\
\tilde{\mathcal{T}} \lambda_{i}^{(4)} \tilde{\mathcal{T}}^{\dagger}=\lambda_{i+1}^{(6)}, & \tilde{\mathcal{T}} \lambda_{i}^{(6)} \tilde{\mathcal{T}}^{\dagger}=\lambda_{i+1}^{(4)}, \\
\tilde{\mathcal{T}} \lambda_{i}^{(3)} \tilde{\mathcal{T}}^{\dagger}=\lambda_{i+1}^{(3)}, & \tilde{\mathcal{T}} \lambda_{i}^{(8)} \tilde{\mathcal{T}}^{\dagger}=\lambda_{i+1}^{(8)} .
\end{aligned}
$$

Since this symmetry involves no change in parity of the photonic operators, no superradiance is involved in a corresponding symmetry-breaking phase transition. It can still be called a parity symmetry, since $\tilde{\mathcal{T}}^{2}=1$, but the parity change involves the qutrit operator $\lambda_{i}^{(3)}$ rather than $\lambda_{i}^{(4)}$ and $\lambda_{i}^{(6)}$ as in Eq. (7). For the spontaneous breaking of the quasitranslation symmetry $\tilde{\mathcal{T}}$, we introduce the order parameter

$$
\varphi \equiv \frac{1}{L} \sum_{i}\left\langle\lambda_{i}^{(3)}\right\rangle
$$

\section{Local gauge symmetries for $s=0$}

In the limit that $s=0$, we find an infinite set of local conserved quantities

$$
\Pi_{i} \equiv \begin{cases}B_{i}^{1} Q_{i} B_{i+1}^{1} & i \text { odd } \\ B_{i}^{2} Q_{i} B_{i+1}^{2} & i \text { even, }\end{cases}
$$

where $Q_{i}=\exp \left(i \pi n_{i}\right)$ and $B_{i}^{k}=\exp \left(i \pi|k\rangle_{i}\left\langle\left. k\right|_{i}\right)(k=1,2)\right.$. We note that the global parity symmetry $\Pi=\bigotimes_{i} \Pi_{i}$ and that $\Pi_{i}$ have the form of generators of a $\mathbb{Z}_{2}$ lattice gauge theory (LGT), where the photons play the role of the "matter field" and the qutrits are bond variables [21]. Gauge theories can lead to interesting phenomena such as confinement, but in one-dimensional quantum systems the gauge theory is "trivial" [33]. The LGT has, however, one immediate consequence. Elitzur's theorem [34] tells us that gauge symmetries can never be broken, and only gauge invariant observables can have nonzero expectation values. E.g., the photon annihilation operator $a_{i}$ is not gauge invariant, and we can predict that $\phi, \phi_{4}, \phi_{6}=0$ when $s=0$, for all values of the light-matter coupling.

\section{E. Effective qutrit Hamiltonian}

It is possible to eliminate the photon degrees of freedom to derive an effective model of interacting $\mathrm{SU}(3)$ spins in one dimension. To obtain such an effective model, we employ the polaron transformation (also called the Lang-Firsov transformation) [35,36], which is an ansatz for the ground state relying on a time-scale separation between fast and slow variables. The method has proven efficient in describing phonon physics, where the states of the ions in a crystal are "displaced" according to the electronic state; phonons (fast variables) dress the electrons (slow variables) to form a polaronic excitation [37]. If the phonons are displaced, the idea is to find the new minima, i.e., the displaced phononic vacuum [36]. The variational ansatz of the polaron transformation for our model is given by

$$
\left|\Psi\left(\left\{c_{i}\right\},\left\{\alpha_{i}\right\}, \gamma\right)\right\rangle=U_{\gamma}^{\dagger}\left|\psi_{\text {qutrit }}\left(\left\{c_{i}\right\}\right)\right\rangle \otimes|\vec{\alpha}\rangle,
$$

where we have defined $|\vec{\alpha}\rangle \equiv \bigotimes_{i}\left|\alpha_{i}\right\rangle$. Here, $\left\{c_{i}\right\}$ are the $3^{N}$ coefficients of the entire many-body state of the qutrits, $\alpha_{i} \in$ $\mathbb{R}$ is the amplitude of a photonic coherent state $\left|\alpha_{i}\right\rangle$, and $\gamma$ is a real parameter which is later chosen such that the photonic and qutrit variables are decoupled. While this is a mean-field ansatz for the photonic variables, it allows for general quantum correlations in the qutrit variables. The unitary polaron transformation is

$$
U_{\gamma} \equiv \exp \left[\gamma \sum_{i}\left(a_{i}-a_{i}^{\dagger}\right) P_{i-1, i}\right],
$$

where we have defined

$$
P_{i-1, i} \equiv \begin{cases}\lambda_{i-1}^{(4)}+\lambda_{i}^{(4)}, & i \text { odd }, \\ \lambda_{i-1}^{(6)}+\lambda_{i}^{(6)}, & i \text { even. }\end{cases}
$$

Taking the expectation value with respect to the photonic variables, $\langle\vec{\alpha}|H| \vec{\alpha}\rangle$, yields the effective qutrit Hamiltonian

$$
\begin{aligned}
H_{\mathrm{eff}}= & -\tilde{s} \sum_{i} \lambda_{i}^{(1)}-\frac{\tilde{\Omega}}{\sqrt{3}} \sum_{i} \lambda_{i}^{(8)} \\
& -J \sum_{i \text { odd }} \lambda_{i-1}^{(4)} \lambda_{i}^{(4)}-J \sum_{i \text { even }} \lambda_{i-1}^{(6)} \lambda_{i}^{(6)},
\end{aligned}
$$

where $J, \tilde{s}$, and $\tilde{\Omega}$ are renormalized couplings. A detailed calculation of Eq. (21), as well as the expressions for the renormalized couplings, are given in Appendix B.

\section{NUMERICAL RESULTS}

\section{A. Matrix product states}

To investigate the possible phases of the system, we have used DMRG to calculate the ground state [38]. DMRG is a variational method with matrix product states (MPSs) as trial states. It can represent gapped, finite-entanglement manybody states to arbitrary accuracy, provided one keeps large enough bond dimension $\chi$ [39]. Even the thermodynamic limit of a translation-invariant system can be described by a MPS representing the unit cell.

Our system can be viewed as a spin model with finite range interactions [40]. For such models, we expect an "area law" behavior of the entropy in gapped phases, i.e., the entropy 


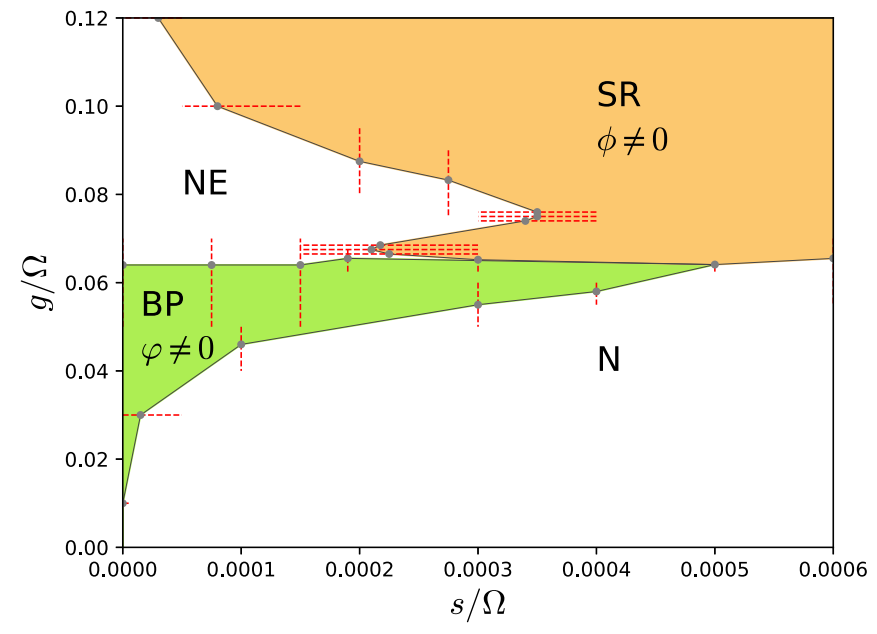

FIG. 2. Schematic phase diagram in the $(s, g)$ plane, calculated with DMRG in the thermodynamic limit for $\omega / \Omega=0.03$. A maximal bond dimension $\chi=100$ was used and the bosonic Hilbert spaces were truncated to dimension 5. The superradiant (SR) phase is characterized by an order parameter $\phi \neq 0$, while the bosonic Peierls (BP) phase is characterized by an order parameter $\varphi \neq 0$. Note that there is no region where both order parameters are nonzero. The normal $(\mathrm{N})$ phase and the normal-entangled (NE) phase are separated in the phase diagram by a multicritical point but are both symmetric phases, having $\phi, \varphi=0$. The sweeps made to calculate the phase boundaries are indicated with dashed red lines.

is proportional to the length of the boundary between the two subsystems, which in one dimension is a single bond. At the critical points, the entanglement is expected to grow logarithmically with subsystem size. In the thermodynamic limit, the entanglement entropy of gapped states should be finite, but diverge as we approach the critical point.

For bosonic degrees of freedom, DMRG requires that we truncate the local Hilbert-space dimension $d$. Increasing $d$ will in principle lead to greater accuracy. However, the computational cost scales as $O\left(\chi^{3} d^{2}\right)$ [41]. We found that expectation values had a strong dependence on $d$ close to critical points, leading to time-consuming calculations. To circumvent this issue, we have used the effective qutrit model (21) to calculate critical exponents. This is justified since the effective qutrit model shares the symmetries of the exact Hamiltonian.

In the paper throughout, we have worked with DMRG directly in the thermodynamic limit, as described above. The Hamiltonian was rescaled as $H / \Omega$ and we have assumed $\omega / \Omega=0.03$. This choice is close to what can be achieved in an implementation with transmon qudits [17].

\section{B. Phase diagram}

In Fig. 2 we show a phase diagram in the $(s, g)$ plane, where the phase boundaries were obtained by sweeping across the boundary and calculating the ground state and order parameters using DMRG, with a maximal bond dimension of $\chi=100$. We verified that choosing a higher value of $\chi$ did not improve the quality of the figure. We find two regions in Fig. 2 where both order parameters are zero, which we call the normal $(\mathrm{N})$ and the normal-entangled (NE) phases. These are separated in the phase diagram by a multicritical
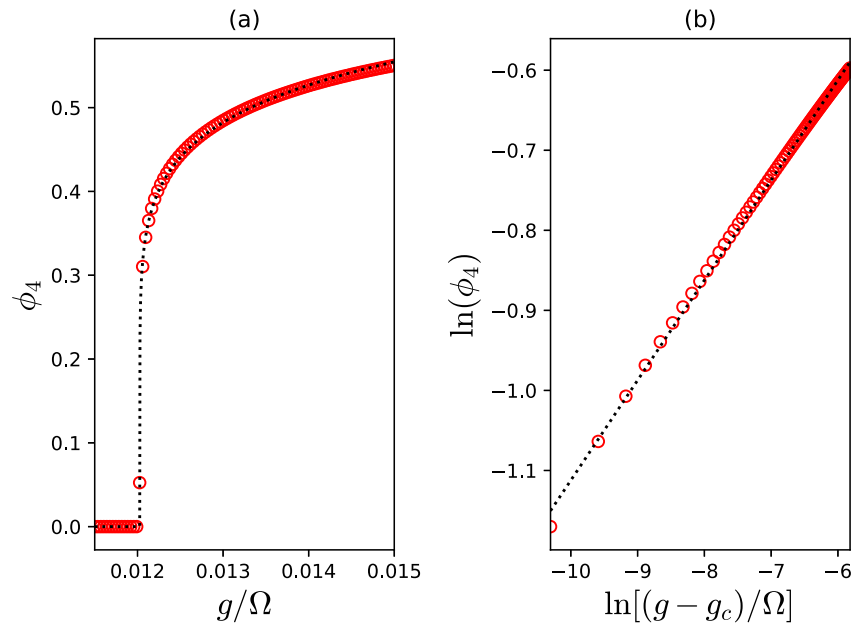

FIG. 3. Plot (a) and log plot (b) of the order parameter (9) over the phase boundary between the $\mathrm{N}$ and SR phases. The circles give the numerical DMRG results, calculated in the thermodynamic limit with a maximal bond dimension $\chi=100$, for the effective qutrit model (21), while the solid lines are curve fits with the exponent $\beta=1 / 8$. The agreement is convincingly good. The remaining parameters were taken as $\omega / \Omega=0.03$ and $s / \Omega=0.2$.

point. There are two regions where the ground state spontaneously breaks symmetries: the superradiant (SR) phase has $\phi, \phi_{4}, \phi_{6} \neq 0$ and thus breaks the parity symmetry (7). We call the phase superradiant because of the aforementioned similarities to the Dicke model. The BP phase has $\varphi \neq 0$ and breaks the quasitranslation symmetry (15). In this phase, every other resonator interacts strongly with its neighboring qutrits, leading to a modulation of period 2 in the density of photons. We find no phase with both symmetries broken simultaneously, i.e., a supersolid. Elitzur's theorem predicts that the SR order parameters cannot be broken, as discussed in Sec. IID. In this sense, the NE phase is to be expected and is connected to the LGT when $s=0$. It is interesting to note that this phase extends to finite $s$, where the gauge theory description does not hold strictly.

As quasitranslation symmetry and the superradiant symmetry are both $\mathbb{Z}_{2}$ symmetries in one spatial dimension, both PTs are expected to fall within the universality class of the transverse-field Ising model in one dimension [11]. We have verified this hypothesis by fitting the magnetization critical exponent $\beta=1 / 8$ to the order parameters calculated using DMRG. As mentioned above, we used the effective qutrit model in these calculations, which is justified when investigating critical features. Figure 3 displays the calculated order parameter (9), tuning $g$ from the $\mathrm{N}$ to the SR phase and keeping $s / \Omega=0.2, \omega / \Omega=0.03$ constant. The data can be fitted to a power law with exponent $\beta=1 / 8$ to excellent agreement. Figure 4 displays the order parameter (16), tuning $g$ from the BP to the NE phase and keeping $s / \Omega=0, \omega / \Omega=0.03$ constant. Again, a power law with exponent $\beta=1 / 8$ is an excellent fit. The symmetry-broken phases SR and BP can be reached from either the $\mathrm{N}$ or the $\mathrm{NE}$ phases, and in all cases we have numerically verified that the exponent $\beta=1 / 8$ is obtained. In all calculations, we found that the MPS and 
(a)

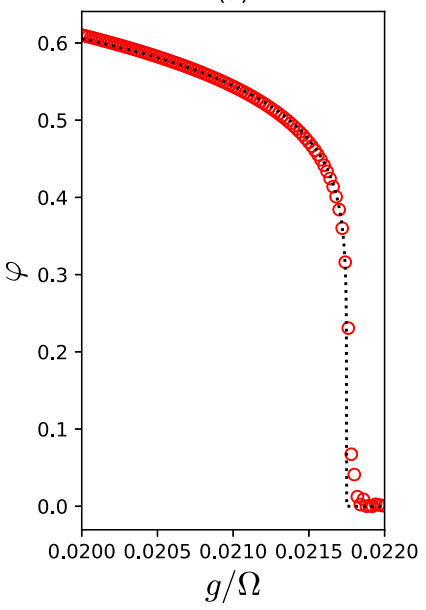

(b)

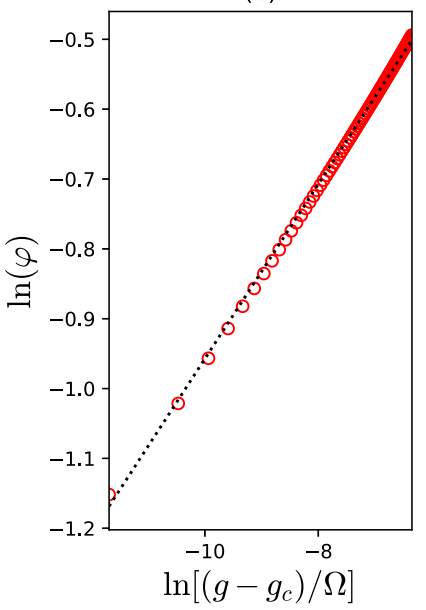

FIG. 4. The same as Fig. 3 but for the order parameter (16) across the phase boundary between the BP and NE phases, with a maximal bond dimension $\chi=100$. Again we find the exponent $\beta=1 / 8$. The other parameters were $\omega / \Omega=0.03$ and $s / \Omega=0$.

expectation values converged for a maximal bond dimension of $\chi=100$.

\section{The bosonic Peierls and normal-entangled phases}

Consider the von Neumann entanglement entropy defined as

$$
S_{\mathrm{vN}} \equiv-\operatorname{Tr}_{1}\left[\rho_{1} \ln \rho_{1}\right],
$$

where $\rho_{1}$ is the reduced density operator of subsystem 1 , obtained by tracing the full density operator over the degrees of freedom of subsystem 2. It is understood that the trace in the above expression is over the degrees of freedom for subsystem 1. In Fig. 5, we plot the entanglement entropy (a) as well as expectation value of the photon number (b) across the BP-NE phase transition for the full model of qutrits and resonators, keeping $s / \omega=0$ and $\omega / \Omega=0.03$. The subsystems 1 and 2 are defined from splitting the full system into two equal halves. In obtaining the figures, the MPS was truncated to a bond dimension of $\chi=100$ and the bosonic Hilbert spaces were truncated to dimension 5, allowing a maximal occupation of four bosons per site. In Fig. 5(a), we see that in the $\mathrm{BP}$ phase, the entanglement is higher between resonators of odd or even index and its adjacent qutrits. The state chooses a higher entanglement around odd or even sites. This staggered structure of the entanglement entropy is another indication of the breaking of the $\mathbb{Z}_{2}$ quasitranslation symmetry. In Fig. 5(b) the photon number also breaks quasitranslation symmetry.

The $\mathrm{N}$ and normal-entangled NE phases are both fully symmetric, and thus considered identical in the Landau classification. They are, however, different in terms of the amount of entanglement entropy (22). Deep in the N phase, $S_{\mathrm{vN}}<$ $10^{-2}$ such that the qutrits and resonators almost form a product state. In the NE phase, however, $S_{\mathrm{vN}} \sim 0.7$ even inside the bulk of the phase, such that the qutrits and resonators are strongly correlated. Even if the N and NE phases share the same global symmetries, there could still exist nonlocal topological order parameters to distinguish them. In the
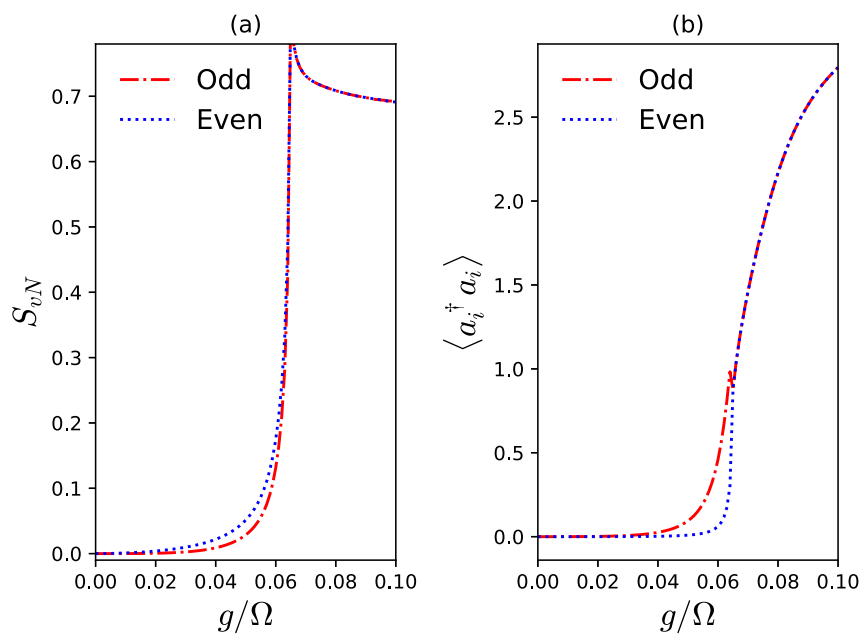

FIG. 5. (a) Entanglement entropy across both the bonds between resonators and qutrits, calculated with DMRG in the thermodynamic limit, for the full Hamiltonian with $s / \Omega=0, \omega / \Omega=0.03$, and a bond dimension $\chi=100$. The bosonic Hilbert spaces were truncated to dimension 5. The entanglement entropy between resonators of odd (even) index and its adjacent qutrits is shown in dashed (dotted) lines. Note that the true entanglement entropy diverges at the critical point, but the chosen entanglement entropy converged for the selected sample points. The plot (b) displays the expectation value of the photon number operator $\left\langle a_{i}^{\dagger} a_{i}\right\rangle$, for odd (even) indices with dashed (dotted) lines, from the same DMRG calculations. The spontaneous breaking of the $\tilde{\mathcal{T}}$ symmetry is evident in the periodic modulation in particle number in (b) and in the staggered entanglement entropy around odd and even resonators in (a).

entanglement structure and MPS description, the NE phase is similar to the Affleck-Kennedy-Lieb-Tasaki (AKLT) state [42], or possibly a valence bond state [43]. The AKLT state has been found to be a symmetry-protected topological state for spin $S$ of odd integer values. One telltale sign of a symmetry-protected topological state is a twofold degeneracy in the entanglement spectrum of the MPS. We have found no such degeneracy in the entanglement spectrum, which indicates that the NE phase is not a symmetry-protected topological state [44].

If the Landau paradigm is not enough to distinguish the $\mathrm{N}$ phase from the NE phase, and no topological [45] feature distinguishes them, there is the possibility that the $\mathrm{N}$ phase could be smoothly deformed into the NE phase. In order to settle the issue, one would need further explorations, e.g., one could try to extract local excitations and determine their properties. For completeness we point out that one could imagine that the vanishing order parameters, $\phi_{4}=0$ and $\varphi=0$, in the NE phase are some numerical artifacts and the two symmetries are indeed broken to give a supersolid. However, this is physically unlikely since for at least $s=0$ we should find an insulator with $\phi=0$ due to the following argument.

Assume no dipolar field on the qutrits, i.e., $s=0$, and further that all qutrits are initially prepared in states $|1\rangle_{i}\left(|2\rangle_{i}\right)$. Then, photons are only able to tunnel between sites $i$ and $i+1$ if $i$ is odd (even), precisely because of the noncommuting structure of the interactions. This "blockade" causes the ground state to be an insulator (the $s=0$ line in the 
phase diagram). We see that these two phases survive for small nonzero coupling values $s$, but for some critical $s_{c}$ the two phases terminate. If the light-matter coupling $g$ is small, the photon number remains small and the system stays an insulator even for large $|s|$ values — the normal phase. In order to find a superfluid state, $g$ has to be increased and the system enters into the SR phase.

\section{PHYSICAL REALIZATION}

The main experimental significance is the selected coupling between a qutrit and its resonators to the left and right. Such selection is easily achieved by adjusting the photon frequencies to become resonant with their respective qutrit transition, but has the disadvantage of leading to a staggered self-energy $\omega$ of the resonators. While the symmetry $\Pi$ connected to the N-SR phase transition survives, such a modification destroys the quasitranslation symmetry $\tilde{\mathcal{T}}$. We have confirmed numerically that the SR phase is robust to such changes $[12,46]$, while unfortunately the BP and NE phases do not survive. Consequently, to access the full phase diagram we need to implement the selection rules by other means. One possibility is to consider external Raman driving between the qutrit levels [47]. There are two advantages with this approach, namely, that the external drive frequency sets the light-matter coupling and it is therefore possible to enter the deep strong-coupling regime, and one can choose to couple the desired transitions. By imposing the rotating-wave approximation, and with the correct frequencies of the resonators and the drive, one circumvents the above-mentioned problem with alternating frequencies of every second resonator (the effective parameter that enters the problem is the detuning which is externally controlled). The resulting Hamiltonian becomes

$$
\begin{aligned}
H_{\mathrm{RWA}}= & \Delta \sum_{i} a_{i}^{\dagger} a_{i}-\frac{\delta}{\sqrt{3}} \sum_{i} \lambda_{i}^{(8)}-s \sum_{i} \lambda_{i}^{(1)}, \\
& +v \sum_{i \text { odd }}\left[a_{i}\left(\lambda_{4, i-1}^{+}+\lambda_{4, i}^{+}\right)+\text {H.c. }\right] \\
& +v \sum_{i \text { even }}\left[a_{i}\left(\lambda_{6, i-1}^{+}+\lambda_{6, i}^{+}\right)+\text {H.c. }\right],
\end{aligned}
$$

where $\Delta$ is the detuning between the corresponding resonator and drive frequencies, $\delta$ is the energy offset of the third level and the lower levels, $v$ is the Raman coupling amplitudes (proportional to the external drive amplitudes), and raising and lowering operators are $\lambda_{6, i}^{( \pm)} \equiv\left(\lambda_{i}^{(4)} \pm \lambda_{i}^{(5)}\right) / 2$ and $\lambda_{6, i}^{( \pm)} \equiv\left(\lambda_{i}^{(6)} \pm \lambda_{i}^{(7)}\right) / 2$. The price one pays is that the rotatingwave approximation alters the symmetries, and thereby also the possible phases [16]. For example, particle conservation (typically arising from the application of the rotating-wave approximation) results in a continuous $U(1)$ symmetry which cannot be broken in one dimension [48]. Nevertheless, a Kosterlitz-Thouless transition is not ruled out by the MerminWagner theorem, and one could still expect phases with superfluidlike properties. We have verified numerically that at least the structure of the phase diagram is the same for the Hamiltonian (23) as that of Fig. 2(b), although the BP phase only survives when the dipolar field strength $s$ is strictly zero.
The largest experimental hindrance is most likely the loss of photons. Two questions arise: how well can we approximate the system by a closed one at short times, and for longer times when losses are significant how is the phase diagram modified? The photon lifetime $\kappa^{-1}$ limits the operation time for any experiment. These typically range between tenths of $\mu$ s to the ms regime. If the setup functions in the deep strongcoupling regime, this lifetime is orders of magnitude longer than typical tunneling times $g^{-1}$, which would allow one to explore the equilibrium phase diagram. However, with current experiments this is a challenge.

The alternative is to actually study nonequilibrium scenarios with coherent photon sources accompanying incoherent photon losses. It is known that the N-SR PT in the Dicke model survives photon losses, even if the universality class is altered [10]. Most likely, also our many-body N-SR PT would survive photon losses. How the superfluid properties are affected by losses has been studied in the past [20]. It was argued that signatures of the equilibrium phase diagram will remain in the driven-dissipative situation, even if novel phenomena may arise [49]. The BP phase is robust and does not depend on photon number conservation, which suggests that it could be verified experimentally. The fate of the NE phase is more subtle since it is a highly entangled state, possibly sensitive to decoherence. However, if the NE phase has topological properties, these can be expected to survive losses.

\section{CONCLUSION}

In this paper, we have studied a generalized Rabi-Hubbard chain, composed by alternating resonators and qutrits in a $\Lambda$ configuration. The qutrits in this setup act both as photon mediators and as a photon nonlinearity. The specific form of the light-matter coupling was chosen to limit the interaction of each resonator to the left (right) leg of its adjacent qutrits. This leads to an insulating bosonic Peierls phase with broken quasitranslational symmetry, which is best understood by considering bosons in a dynamical lattice of the qutrits. The inclusion of the dipole field opens up for a superradiant phase. The transition from the normal insulating phase to this superfluid phase can be identified with a truly many-body normal-superradiant QPT, contrary to the one of the Dicke model which is of the mean-field type. Despite the presence of the BP and SR phases, we do not find numerical evidence for a supersolid.

The simplest scheme in order to realize the qutrit-resonator setup would be to consider Raman transitions driven by external classical fields. This, however, is typically analyzed within the rotating-wave approximation. The effect of omitting the counter-rotating terms is interesting and left for the future. In our analysis we did not take losses into account, meaning that the results are strictly only experimentally relevant for time scales shorter than the photon and qutrit lifetimes. However, the findings are still experimentally justifiable since the physics of the closed system is believed to leave traces in the driven open system [20], i.e., the photon blockade effect is known to exist also for driven-dissipative systems. Nevertheless, a drive combined with losses might give additional interesting features not captured in our paper. 
We end by mentioning the idea of studying similar q-dot generated photon tunneling in two dimensions. Extending an experiment in this way should not be too difficult. In higher dimensions the lattice geometry may play an important roleone can imagine a plethora of different settings like lattices supporting flat bands, confinement, Dirac cones [50], or other more exotic lattices [51].

\section{ACKNOWLEDGMENTS}

We thank Chitanya Joshi, Stefan Filipp, Iman Mahyaeh, Pil Saugmann, and Themistoklis Mavrogordatos for helpful discussions. We acknowledge financial support from the Wallenberg Academy Fellows program of the Knut and Alice Wallenberg Foundation and the Swedish Research Council.

\section{APPENDIX A: GELL-MANN MATRICES}

Just as the Pauli matrices are generators of SU(2), the Gell-Mann matrices $\lambda^{(i)}(i=1,2, \ldots, 8)$ are generators of $\mathrm{SU}(3)$; i.e., they are Hermitian, traceless $\left[\operatorname{Tr}\left(\lambda^{(i)}\right)=0\right]$, and orthogonal $\left[\operatorname{Tr}\left(\lambda^{(i)} \lambda^{(j)}\right)=2 \delta_{i j}\right.$ ] [29]. It should be clear that together with the identity matrix, any complex $3 \times 3$ matrix can be expressed as a linear combination of the $\lambda^{(i)}$ matrices. The usual representation of the Gell-Mann matrices is

$$
\begin{array}{ll}
\lambda^{(1)}=\left(\begin{array}{lll}
0 & 1 & 0 \\
1 & 0 & 0 \\
0 & 0 & 0
\end{array}\right), & \lambda^{(2)}=\left(\begin{array}{rrr}
0 & -i & 0 \\
i & 0 & 0 \\
0 & 0 & 0
\end{array}\right), \\
\lambda_{3}=\left(\begin{array}{rrr}
1 & 0 & 0 \\
0 & -1 & 0 \\
0 & 0 & 0
\end{array}\right), & \lambda^{(4)}=\left(\begin{array}{lll}
0 & 0 & 1 \\
0 & 0 & 0 \\
1 & 0 & 0
\end{array}\right), \\
\lambda^{(5)}=\left(\begin{array}{rrr}
0 & 0 & -i \\
0 & 0 & 0 \\
i & 0 & 0
\end{array}\right), & \lambda^{(6)}=\left(\begin{array}{lll}
0 & 0 & 0 \\
0 & 0 & 1 \\
0 & 1 & 0
\end{array}\right), \\
\lambda^{(7)}=\left(\begin{array}{rrr}
0 & 0 & 0 \\
0 & 0 & -i \\
0 & i & 0
\end{array}\right), & \lambda^{(8)}=\frac{1}{\sqrt{3}}\left(\begin{array}{rrr}
1 & 0 & 0 \\
0 & 1 & 0 \\
0 & 0 & -2
\end{array}\right) .
\end{array}
$$

Note that the Gell-Mann matrices can be written as products of matrices of the spin-1 representation of SU(2). However, only by using the Gell-Mann matrices can Eq. (1) of the main text be written as a quadratic Hamiltonian.

\section{APPENDIX B: POLARON ANSATZ}

The goal of this Appendix is to calculate the effective qutrit Hamiltonian (21), which is found by taking the expectation value with respect to the photonic variables only, i.e.,

$$
H_{\text {eff }}=\langle\vec{\alpha}|H| \vec{\alpha}\rangle,
$$

where, as in the main text, we have defined $|\vec{\alpha}\rangle \equiv \bigotimes_{i}\left|\alpha_{i}\right\rangle$. It is useful to write the polaron unitary $U_{\gamma}$ as follows:

$$
\begin{aligned}
U_{\gamma} & =\exp (-i \gamma S), \\
S & =\sum_{i} i\left(a_{i}-a_{i}^{\dagger}\right) P_{i-1, i}=\sum_{i} S_{i, i+1},
\end{aligned}
$$

where we have defined the two-site operators

$$
P_{i-1, i} \equiv \begin{cases}\lambda_{i-1}^{(4)}+\lambda_{i}^{(4)} & i \text { odd } \\ \lambda_{i-1}^{(6)}+\lambda_{i}^{(6)} & i \text { even }\end{cases}
$$

and

$$
\begin{aligned}
& S_{i, i+1}= \begin{cases}-\sqrt{2}\left(p_{i} \lambda_{i}^{(4)}+\lambda_{i}^{(6)} p_{i+1}\right) & i \text { odd, } \\
-\sqrt{2}\left(p_{i} \lambda_{i}^{(6)}+\lambda_{i}^{(4)} p_{i+1}\right) & i \text { even, }\end{cases} \\
& p_{i}=-\frac{i}{\sqrt{2}}\left(a_{i}-a_{i}^{\dagger}\right) .
\end{aligned}
$$

Note that both $P_{i-1, i}$ and $S_{i, i+1}$ are unitary, both involve operators from two sites, and $\left[P_{i-1, i}, P_{i, i+1}\right] \neq 0$ and $\left[S_{i-1, i}, S_{i, i+1}\right] \neq 0 . S_{i, i+1}$ only involves qutrit operators for one site, while $\left(a_{i}-a_{i}^{\dagger}\right) P_{i-1, i}$ only involves photonic operators from one site.

$U_{\gamma}$ acts like a displacement operator on the photonic variables, i.e.,

$$
U_{\gamma} a_{i} U_{\gamma}^{\dagger}=a_{i}+\gamma\left[a_{i}-a_{i}^{\dagger}, a_{i}\right] P_{i-1, i}=a_{i}+\gamma P_{i-1, i},
$$

which allows us to calculate the contribution to $H_{\text {eff }}$ from the photonic part of $H_{B}$ immediately as

$$
\sum_{i}\left\langle\vec{\alpha}\left|U_{\gamma} a_{i}^{\dagger} a_{i} U_{\gamma}^{\dagger}\right| \vec{\alpha}\right\rangle=\sum_{i}\left(\alpha_{i}^{2}+2 \gamma \alpha_{i} P_{i-1, i}+\gamma^{2} P_{i-1, i}^{2}\right) .
$$

The transformation of the qutrit part of $H_{B}$ is more involved. Using the Hadamard lemma [52], the transformed qutrit operators can be expanded as power series of nested commutators as

$$
\begin{aligned}
U_{\gamma} \lambda_{i}^{(\alpha)} U_{\gamma}^{\dagger}= & \lambda_{i}^{(\alpha)}-i \gamma\left[S_{i, i+1}, \lambda_{i}^{(\alpha)}\right] \\
& +\frac{(-i \gamma)^{2}}{2 !}\left[S_{i, i+1},\left[S_{i, i+1}, \lambda_{i}^{(\alpha)}\right]\right]+\cdots .
\end{aligned}
$$

To compute coherent-state expectation values, we use the formula (assuming $\alpha \in \mathbb{R}$ )

$$
\left\langle\alpha\left|p^{n}\right| \alpha\right\rangle= \begin{cases}0 & n \text { odd } \\ \Gamma\left(\frac{n+1}{2}\right) / \sqrt{\pi} & n \text { even. }\end{cases}
$$

Using (B8), we find

$$
\begin{gathered}
-s \sum_{i}\left\langle\vec{\alpha}\left|U_{\gamma} \lambda_{i}^{(1)} U_{\gamma}^{\dagger}\right| \vec{\alpha}\right\rangle=-s f_{1}(\gamma) \sum_{i} \lambda_{i}^{(1)} \\
-\frac{\Omega}{\sqrt{3}} \sum_{i}\left\langle\vec{\alpha}\left|U_{\gamma} \lambda_{i}^{(8)} U_{\gamma}^{\dagger}\right| \vec{\alpha}\right\rangle=-\frac{\Omega}{\sqrt{3}} f_{8}(\gamma) \sum_{i} \lambda_{i}^{(8)},
\end{gathered}
$$

where the functions of $\gamma$ can be expanded as rapidly converging power series

$$
\begin{aligned}
& f_{1}(\gamma)=1-\gamma^{2}+\frac{5 \gamma^{4}}{6}-\frac{17 \gamma^{6}}{30}+O\left(\gamma^{8}\right), \\
& f_{8}(\gamma)=1-3 \gamma^{2}+4 \gamma^{4}-\frac{16 \gamma^{6}}{5}+O\left(\gamma^{8}\right) .
\end{aligned}
$$


It remains to calculate the contribution from the interaction Hamiltonian:

$$
\begin{aligned}
& g \sum_{i}\left\langle\vec{\alpha}\left|U_{\gamma}\left(a_{i}+a_{i}^{\dagger}\right) U_{\gamma}^{\dagger} U_{\gamma} P_{i-1, i} U_{\gamma}^{\dagger}\right| \vec{\alpha}\right\rangle \\
& =g \sum_{i}\left\langle\vec{\alpha}\left|\left(a_{i}+a_{i}^{\dagger}\right) U_{\gamma} P_{i-1, i} U_{\gamma}^{\dagger}\right| \vec{\alpha}\right\rangle \\
& \quad+2 g \gamma \sum_{i} P_{i-1, i}\left\langle\vec{\alpha}\left|U_{\gamma} P_{i-1, i} U_{\gamma}^{\dagger}\right| \vec{\alpha}\right\rangle .
\end{aligned}
$$

In the calculation, we will need to use the formula

$$
\left\langle\alpha\left|\left(a+a^{\dagger}\right) p^{n}\right| \alpha\right\rangle= \begin{cases}i n / \sqrt{2}\left\langle\alpha\left|p^{n-1}\right| \alpha\right\rangle & n \text { odd } \\ 2 \alpha\left\langle\alpha\left|p^{n}\right| \alpha\right\rangle & n \text { even. }\end{cases}
$$

Using (B12), we find

$$
\begin{aligned}
& g \sum_{i}\left\langle\vec{\alpha}\left|\left(a_{i}+a_{i}^{\dagger}\right) U_{\gamma} P_{i-1, i} U_{\gamma}^{\dagger}\right| \vec{\alpha}\right\rangle \\
& =g \sum_{i \text { odd }} \sum_{\beta=2,4,6}\left(h_{i-1, i}^{4 \beta} \lambda_{i-1}^{\beta}+h_{i, i+1}^{4 \beta} \lambda_{i}^{\beta}\right) \\
& \quad+g \sum_{i \text { even } \beta=2,4,6}\left(h_{i-1, i}^{6 \beta} \lambda_{i-1}^{\beta}+h_{i, i+1}^{6 \beta} \lambda_{i}^{\beta}\right) \\
& =g f_{g}(\gamma) \sum_{i} 2 \alpha_{i} P_{i-1, i},
\end{aligned}
$$

since $h_{i-1, i}^{64}=h_{i-1, i}^{46}=0$ for all $i, h_{i, i+1}^{42}=h_{i-1, i}^{62}=0$ and $h_{i, i+1}^{42}=-h_{i-1, i}^{62}$ for $i$ odd and vice versa for $i$ even, while $h_{i, i+1}^{66}=h_{i, i+1}^{44}=f_{g}(\gamma)$ for all $i$, where

$$
f_{g}(\gamma)=1-\frac{\gamma^{2}}{2}+\frac{\gamma^{4}}{6}-\frac{\gamma^{6}}{30}+\frac{\gamma^{8}}{210}+O\left(\gamma^{10}\right) .
$$

Note that the denominators are the primorial numbers [53], so $f_{g}(\gamma)$ is the "primorial version" of $e^{-\gamma^{2}}$.

Furthermore, from (B8), we know that only coherent-state expectation values of even powers of $p^{n}$ are nonzero. By expanding in a power series using the Hadamard lemma and setting all terms which are odd in $p_{i-1}$ or $p_{i}$ to zero, we find

$$
2 g \gamma \sum_{i} P_{i-1, i}\left\langle\vec{\alpha}\left|U_{\gamma} P_{i-1, i} U_{\gamma}^{\dagger}\right| \vec{\alpha}\right\rangle=2 g \gamma f_{g}(\gamma) \sum_{i} P_{i-1, i}^{2} .
$$

The expression (B13) and the cross-term in (B6) are the only terms which couple the photonic mean-field and qutrit degrees of freedom. Both terms can be eliminated by choosing $\gamma$ to be a root of the equation

$$
g f_{g}(\gamma)=-\omega \gamma
$$

which has a solution $\gamma \sim-g / \omega$ close to $g / \omega=0$. By this choice, the photonic and qutrit degrees of freedom are completely decoupled. Once decoupled, the photonic degrees of freedom can be minimized independently to $\alpha_{i}=0$. In this sense, the polaron ansatz is a "dynamic displacement" of the photonic vacuum. The ansatz neglects any contribution to quantum fluctuations from the resonators around this vacuum state.

We found that the photonic part of the effective qutrit Hamiltonian was minimized to zero, so the remaining part is a qutrit Hamiltonian:

$$
\begin{aligned}
H_{\mathrm{eff}}= & -s f_{1}(\gamma) \sum_{i} \lambda_{i}^{(1)}-\frac{\Omega}{\sqrt{3}} f_{8}(\gamma) \sum_{i} \lambda_{i}^{(8)} \\
& +\left[\omega \gamma^{2}+2 g \gamma f_{g}(\gamma)\right] \sum_{i} P_{i-1, i}^{2} .
\end{aligned}
$$

Assuming periodic boundary conditions, we find (the constant term is dropped)

$$
\begin{aligned}
\sum_{i} P_{i-1, i}^{2}= & \sum_{i \text { odd }}\left[\left(\lambda_{i-1}^{(4)}\right)^{2}+\left(\lambda_{i-1}^{(4)}\right)^{2}+2 \lambda_{i-1}^{(4)} \lambda_{i}^{(4)}\right] \\
& +\sum_{i \text { even }}\left[\left(\lambda_{i-1}^{(6)}\right)^{2}+\left(\lambda_{i-1}^{(6)}\right)^{2}+2 \lambda_{i-1}^{(6)} \lambda_{i}^{(6)}\right] \\
= & 2 \sum_{i \text { odd }} \lambda_{i-1}^{(4)} \lambda_{i}^{(4)}+2 \sum_{i \text { even }} \lambda_{i-1}^{(6)} \lambda_{i}^{(6)} \\
& +\sum_{i}\left(\frac{4}{3} \mathbb{I}_{3 \times 3}-\frac{1}{\sqrt{3}} \lambda_{i}^{(8)}\right),
\end{aligned}
$$

such that the effective qutrit Hamiltonian can be written as

$$
\begin{aligned}
H_{\mathrm{eff}}= & -\tilde{s} \sum_{i} \lambda_{i}^{(1)}-\frac{\tilde{\Omega}}{\sqrt{3}} \sum_{i} \lambda_{i}^{(8)} \\
& -J \sum_{i \text { odd }} \lambda_{i-1}^{(4)} \lambda_{i}^{(4)}-J \sum_{i \text { even }} \lambda_{i-1}^{(6)} \lambda_{i}^{(6)},
\end{aligned}
$$

where

$$
\begin{aligned}
\tilde{s} & =f_{1}(\gamma) s, \\
\tilde{\Omega} & =f_{8}(\gamma) \Omega+J / 2, \\
J & =-2\left[\omega \gamma^{2}+2 g \gamma f_{g}(\gamma)\right]=2 \omega \gamma^{2} .
\end{aligned}
$$

[1] I. Carusotto and C. Ciuti, Rev. Mod. Phys. 85, 299 (2013).

[2] V. DeGiorgio and M. O. Scully, Phys. Rev. A 2, 1170 (1970); H. Haken, Laser Theory (Springer-Verlag, Berlin, 2012).

[3] R. H. Dicke, Phys. Rev. 93, 99 (1954).

[4] B. M. Garraway, Phil. Trans. R. Soc. A 369, 1137 (2011).

[5] K. Hepp and E. H. Lieb, Ann. Phys. (NY) 76, 360 (1973).

[6] Y. K. Wang and F. T. Hioe, Phys. Rev. A 7, 831 (1973).

[7] M. J. Hartmann, F. G. S. L. Brandao, and M. B. Plenio, Laser Photonics Rev. 2, 527 (2008).

[8] J. I. Cirac and P. Zoller, Nat. Phys. 8, 264 (2012).
[9] M. Hillery and L. D. Mlodinow, Phys. Rev. A 31, 797 (1985).

[10] J. Larson and E. K. Irish, J. Phys. A: Math. Gen. 50, 17002 (2017).

[11] S. Sachdev, Quantum Phase Transition (Cambridge University, Cambridge, England, 2011).

[12] P. Strack and S. Sachdev, Phys. Rev. Lett. 107, 277202 (2011); M. Buchhold, P. Strack, S. Sachdev, and S. Diehl, Phys. Rev. A 87, 063622 (2013).

[13] M. J. Hartmann, F. G. S. L. Brandao, and M. B Plenio, Nat. Phys. 2, 849 (2006); A. D. Greentree, C. Tahan, J. H-Cole, and 
L. C. L. Hollenberg, ibid. 2, 856 (2006); A. Tomadin and R. Fazio, J. Opt. Soc. Am. B 27, A130 (2010); S. Schmidt and J. Koch, Ann. Phys. (NY) 525, 395 (2013); S. Schmidt, G. Blatter, and J. Keeling, J. Phys. B 46, 224020 (2013).

[14] A. Wallraff, D. I. Schuster, A. Blais, L. Frunzio, R.- S. Huang, J. Majer, S. Kumar, S. M. Girvin, and R. J. Schoelkopf, Nature (London) 431, 162 (2004).

[15] K. M. Birnbaum, A. Boca, R. Miller, A. D. Boozer, T. E. Northup, and H. J. Kimble, Nature (London) 436, 87 (2005).

[16] In the Jaynes-Cummings-Hubbard model, the particle number is preserved and it belongs to the same universality class as the Bose-Hubbard model. Without the rotating-wave approximation, i.e., the Rabi-Hubbard model, the particle number is no longer preserved but it supports a parity symmetry and it belongs instead to the Ising universality class; M. Schiro, M. Bordyuh, B. Öztop, and H. E. Türeci, Phys. Rev. Lett. 109, 053601 (2012).

[17] A. Kurcz, A. Bermudez, and J. J. García-Ripoll, Phys. Rev. Lett. 112, 180405 (2014).

[18] C. P. Sun, L. F. Wei, Y. X. Liu, and F. Nori, Phys. Rev. A 73, 022318 (2006); M. Mariantoni, F. Deppe, A. Marx, R. Gross, F. K. Wilhelm, and E. Solano, Phys. Rev. B 78, 104508 (2008).

[19] M. Mariantoni, H. Wang, R. C. Bialczak, M. Lenander, E. Lucero, M. Neeley, A. D. O'Connell, D. Sank, M. Weides, J. Wenner, T. Yamamoto, Y. Yin, J. Zhao, J. M. Martinis, and A. N. Cleland, Nat. Phys. 7, 287 (2011).

[20] A. Tomadin, V. Giovannetti, R. Fazio, D. Gerace, I. Carusotto, H. E. Türeci, and A. Imamoglu, Phys. Rev. A 81, 061801(R) (2010); F. Nissen, S. Schmidt, M. Biondi, G. Blatter, H. E. Türeci, and J. Keeling, Phys. Rev. Lett. 108, 233603 (2012); J. Jin, D. Rossini, R. Fazio, M. Leib, and M. J. Hartmann, ibid. 110, 163605 (2013).

[21] U.-J. Wiese, Ann. Phys. (NY) 525, 777 (2013); D. Marcos, P. Rabl, E. Rico, and P. Zoller, Phys. Rev. Lett. 111, 110504 (2013); P. Hauke, D. Marcos, M. Dalmonte, and P. Zoller, Phys. Rev. X 3, 041018 (2013).

[22] R. Peierls, Quantum Theory of Solids (Clarendon, Oxford, 1955).

[23] R. E. Peierls, Quantum Theory of Solids (Oxford University, London, 1955).

[24] J. Léonard, A. Morales, P. Zupancic, T. Esslinger, and T. Donner, Nature (London) 543, 87 (2017).

[25] D. González-Cuadra, P. R. Grzybowski, A. Dauphin, and M. Lewenstein, Phys. Rev. Lett. 121, 090402 (2018).

[26] I. Shomroni, S. Rosenblum, Y. Lovsky, O. Bechler, G. Guendelman, and B. Dayan, Science 345, 903 (2014).

[27] S. Rosenblum, O. Bechler, I. Shomroni, Y. Lovsky, G. Guendelman, and B. Dayan, Nature Phot. 10, 19 (2016).

[28] J. Casanova, G. Romero, I. Lizuain, J. J. Garcia-Ripoll, and E. Solano, Phys. Rev. Lett. 105, 263603 (2010).
[29] H. Georgi, Lie Algebras in Particle Physics (Westview, Boulder, 1999).

[30] M. Greiter and S. Rachel, Phys. Rev. B 75, 184441 (2007).

[31] N. Goldenfeld, Lectures On Phase Transitions And The Renormalization Group (Addison-Wesley, Reading, MA, 1992).

[32] K. Baumann, C. Guerlin, F. Brennecke, and T. Esslinger, Nature (London) 464, 1301 (2010); M. P. Baden, K. J. Arnold, A. L. Grimsmo, S. Parkins, and M. D. Barrett, Phys. Rev. Lett. 113, 020408 (2014).

[33] J. B. Kogut, Rev. Mod. Phys. 4, 51 (1979).

[34] S. Elitzur, Phys. Rev. D 12, 3978 (1975).

[35] G. Lang and Y. A. Firsov, Zh. Eksp. Teor. Fiz. 43, 1843 (1962).

[36] G. D. Mahan, Many-Particle Physics (Plenum, New York, 1990), Chap. 4; A. Würger, Phys. Rev. B 57, 347 (1998).

[37] G. D. Mahan, Many-Particle Physics, 3rd ed. (Kluwer Academic, New York, 2000).

[38] J. Hauschild and F. Pollmann, SciPost Phys. Lect. Notes (2018), code available from https://github.com/tenpy/tenpy.

[39] U. Schollwöck, Ann. Phys. (NY) 326, 96 (2011).

[40] J. Eisert, M. Cramer, and M. B. Plenio, Rev. Mod. Phys. 82, 277 (2010).

[41] E. Jeckelmann and H. Fehske, Riv. Nuovo Cimento 6, 30 (2007).

[42] I. Affleck, T. Kennedy, E. H. Lieb, and H. Tasaki, Phys. Rev. Lett. 59, 799 (1987).

[43] X.-G. Wen, Quantum Field Theory of Many-Body Systems (Oxford University, London, 2004)

[44] M. Oshikawa, J. Phys.: Condens. Matter 4, 36 (1992); F. Pollmann, E. Berg, A. M. Turner, and M. Oshikawa, Phys. Rev. B 85, 075125 (2012).

[45] X. Chen, Z.-C. Gu, and X.-G. Wen, Phys. Rev. B 83, 035107 (2011)

[46] L.-J. Zou, D. Marcos, S. Diehl, S. Putz, J. Schmiedmayer, J. Majer, and P. Rabl, Phys. Rev. Lett. 113, 023603 (2014).

[47] F. Dimer, B. Estienne, A. S. Parkins, and H. J. Carmichael, Phys. Rev. A 75, 013804 (2007).

[48] A. Auerbach, Interacting Electrons and Quantum Magnetism (Springer, New York, 2012).

[49] A. Le Boité, G. Orso, and C. Ciuti, Phys. Rev. Lett. 110, 233601 (2013)

[50] A. A. Houch, H. E. Türeci, and J. Koch, Nat. Phys. 8, 292 (2012)

[51] A. J. Kollar, M. Fitzpatrick, and A. A. Houck, Nature (London) 571, 45 (2019); A. J. Kollár, M. Fitzpatrick, P. Sarnak, and A. A. Houck, Commun. Math. Phys. 376, 1909 (2020).

[52] R. Shankar, Principles of Quantum Mechanics (Plenum, New York, 1994).

[53] H. Dubner, J. Recreat. Math. 19, 197 (1987). 